\title{
Properties of Additive-Manufactured Open Porous Titanium Structures for Patient-Specific Load-Bearing Implants
}

\author{
Livia Zumofen ${ }^{1 *}$ Katarzyna S. Kopanska ${ }^{2}$, Epifania Bono ${ }^{2}$, Andreas Kirchheim ${ }^{1}$, \\ Emmanuel B. De Haller ${ }^{3}$ and Ursula Graf-Hausner ${ }^{2}$
}

${ }^{1}$ Centre for Product and Process Development ZPP, School of Engineering, Zurich University of Applied Sciences ZHAW, Winterthur, Switzerland, ${ }^{2}$ Institute of Chemistry and Biotechnology ICBT, Life Science and Facility Management, Zurich University of Applied Sciences ZHAW, Waedenswil, Switzerland, ${ }^{3}$ Nobel Biocare, Kloten, Switzerland

OPEN ACCESS

Edited by:

Gang Jl,

National Centre for Scientific

Research, France

Reviewed by:

Amir Putra Md Saad,

Universiti Teknologi Malaysia (UTM),

Malaysia

Yongtao Lu,

Dalian University of Technology (DUT),

China

*Correspondence:

Livia Zumofen

livia.zumofen@zhaw.ch

Specialty section:

This article was submitted to

Digital Manufacturing,

a section of the journal

Frontiers in Mechanical Engineering

Received: 06 December 2021

Accepted: 28 December 2021

Published: 01 February 2022

Citation:

Zumofen L, Kopanska KS, Bono E,

Kirchheim A, De Haller EB and Graf-Hausner U (2022) Properties of Additive-Manufactured Open Porous

Titanium Structures for Patient-

Specific Load-Bearing Implants.

Front. Mech. Eng 7:830126.

doi: 10.3389/fmech.2021.830126
Additive manufacturing has been well established in many sectors, including the medical industry. For load-bearing bone implants, titanium and its alloys, such as Ti6Al4V, are widely used due to their high strength to weight ratio and osseointegrative properties. However, bone resorption and loosening of implants is related to the significantly higher stiffness of dense Ti6AI4V, leading to stress shielding. With the aging of population, there is an increasing need for orthopedic implants with a high success rate and a long implant life span. Besides that the treatment of non-healing segmental bone defects, where the selfrepairing properties of bone tissue are not sufficient, is still a challenge. In both fields of application, patient-specific titanium implants combined with functionally graded porosity designed according to locally expected loads unlock new possibilities. Many studies underline the huge potential of the new design freedom to generate open porous structures and more personalized implants with enhanced mechanical properties that also integrate well with surrounding tissues. Integration of functionally graded open porosity into implants allows for the implant to more closely mimic the mechanical properties of human bone and its internal architecture. The results of this work represent the basis for developing complex porous titanium structures with various pore sizes and shapes to tailor structural mechanical properties and biological responses. Therefore, $3 \mathrm{D}$ porous structures with various pore sizes and shapes were designed and manufactured in Ti6AI4V using laser powder bed fusion (PBF-LB/M). Based on these structures, the correlation of pore size and shape with cell ingrowth, morphology, metabolic activity, and early markers for bone formation (ALP activity) was investigated in static cell cultures using the osteosarcoma cell line Saos-2. Mechanical properties, such as stiffness and compression strength, were investigated with compression testing. The present study concludes that cell morphology, metabolic activity, and ALP activity are widely independent of pore shape and size within the tested range of 400-700 $\mu \mathrm{m}$ pore size. Furthermore, the mechanical properties of the evaluated structures were in the range of cortical and trabecular bone. This opens the possibility to design mechanical properties with gradient porosity without decisively affecting biological responses.

Keywords: 3D printing, additive manufacturing, porous titanium, 3D cell cultures, implant, PBF-LB/M, laser melting, biomaterial 


\section{INTRODUCTION}

Bone displays a high intrinsic regenerative potential. Minor defects often heal spontaneously within several weeks or months. However, severe bone losses, for example, from different pathologies or accidents, lead to the "criticalsized" bone defect (CSD), which is defined as a defect of a size that will not heal during a lifetime without intervention (Schmitz and Hollinger, 1986). In particular, very large CSDs that require bone grafting to fill the defect present a major challenge. Currently used strategies have several limitations. Bone transplants, most commonly used in practice, have very limited availability. Biocompatible natural or synthetic implant materials, on the other hand, offer an alternative, but to-date none of them meet the requirements to match the native bone (Gugala et al., 2007). Challenges remain to adjust the material to the specific mechanical properties of the bone: viscoelasticity, anisotropy, and unique high toughness, stiffness, and specific strength compared to other tissues (Alvarez and Nakajima, 2009). Moreover, all these factors are unique for each bone type and depend on the subject's condition, age, anatomic location, and on specific load application (Albrektsson and Johansson, 2001; Parthasarathy et al., 2011). This high level of variation is already present within the same bone, in cortical and trabecular compartments. The cortical bone exhibits a low porosity of 5-10\% and a stiffness of 14-30 GPa. The trabecular bone, on the other hand, is characterized by high porosity of $50-90 \%$ and a stiffness of $0.3-18 \mathrm{GPa}$ (Rho et al., 1998; Morgan and Keaveny, 2001; Geetha et al., 2009) (Figure 1A). Therefore, the flexibility in matching the mechanical and structural features and the overall shape of the reconstructed area are critical for the successful treatment of large patient-specific CSDs.

The most widely used materials in bone repair are based on minerals (natural or synthetic), such as natural hydroxyapatite or ceramics (Henkel et al., 2013). Such materials mostly have good osteoconductive properties and promote bone regeneration, but lack tensile strength for initial load-bearing and primary stability, limiting their application to non-load-bearing situations or requiring long periods of immobilization during bone healing (Henkel et al., 2013). This represents a major drawback, as early stress stimulation is imperative for efficient long-term bone healing (Klosterhoff et al., 2020).

Titanium and titanium alloys provide attractive alternatives to mineral bone substitute materials and have been frequently used in dental and orthopedic surgery (Alvarez and Nakajima, 2009). Inert in the human body environment, titanium offers a high strength-to-weight ratio, good fatigue resistance in biological media, and low stiffness compared to other metals such as stainless steel and cobalt-chromium alloys (CoCr) (Figure 1B) (Geetha et al., 2009). Compared to commercially pure titanium, the alloy Ti6Al4V exhibits approximately threefold tensile strength and enhanced fatigue resistance. Therefore, Ti6Al4V is the most widely used titanium alloy for medical devices (Niinomi, 1998; Geetha et al., 2009). However, Ti6Al4V has a much higher stiffness than human trabecular and cortical bone, $114 \mathrm{GPa}$ and $0.4-30 \mathrm{GPa}$, respectively (Niinomi, 1998; Rho et al., 1998; Morgan and Keaveny, 2001; Geetha et al., 2009). Such a mismatch in stiffness of implant and host-tissue leads to an unfavorable stress distribution at the bone implant interface, called stress shielding, which may ultimately lead to bone resorption and loosening of the implant.

The mechanical properties of three-dimensional (3D) structures can be described in terms of structural and material properties analogous to those of trabecular bone. Structural properties are defined as the overall properties of both metal trabecular struts and pores. In contrast, material properties are defined as the intrinsic properties of the Ti6Al4V alloy itself. Structural stiffness of titanium implants can be reduced by increasing porosity to get closer to the host-tissue properties (Geetha et al., 2009; Parthasarathy et al., 2011).

\begin{tabular}{lll}
\hline & & \\
\hline Human cortical bone & $\begin{array}{l}\text { Porosity } \\
{[\%]}\end{array}$ & $\begin{array}{l}\text { Young's modulus } \\
{[\mathrm{GPa}]}\end{array}$ \\
\hline Human trabecular bone & $50-10$ & $14-30$ \\
\hline
\end{tabular}

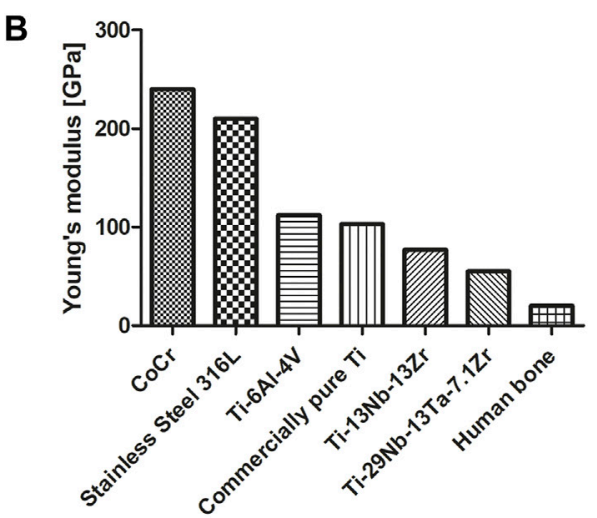

FIGURE 1 | (A) Young's modulus of cortical and trabecular bone [adapted from (Schoenfeld et al., 1974; Rho et al., 1998; Misch et al., 1999; Keaveny et al., 2004; Geetha et al., 2009; Cordonnier et al., 2011; Hoffmann et al., 2014)]; and (B) different metals and metal alloys currently used for medical implants [adapted from (Niinomi, 1998; Geetha et al., 2009)]. 
There are numerous ways to design open porous structures, including parametric and non-parametric design approaches. Non-parametric design is the more classical approach, which is focused on structural and geometric design. Parametric designs rely on cellular structures that are generated based on specific algorithms (Chen et al., 2020). The Voroni structure is an open, porous mesh structure that is based on the connection of random discrete points to form a network (Liu et al., 2019). It's seen as a highly attractive design method for orthopedic implants, as it offers a similar structure to the morphology of bone (Chen et al., 2020). Although parametric design methods are highly interesting to generate more complex porous structures and gradient porosity with nonuniform pore sizes and shapes, they also have their limitations. To study the cell biological response in relation to specific pore characteristics, such as size and shape, the investigated structures require a uniform size and shape throughout the structure.

Laser powder bed fusion (PBF-LB/M) is an emerging additive manufacturing (AM) technology that enables the layer-wise generation of complex metal structures. The layer-wise principle and the direct link to a $3 \mathrm{D}$ model result in new design freedom and simplification of product customization (Gibson et al., 2021). Therefore, PBF-LB/M unlocks new possibilities in the design and manufacturing of medical implants for enhanced fit, performance, and personalization. For example, PBF-LB/M of titanium structures offers very high flexibility in terms of shape and structure of the implant and, most importantly, in terms of mechanical properties, by the introduction of the appropriate open porosity with controlled architecture (Lewis, 2013; ElHajje et al., 2014; Ataee et al., 2018; Chen et al., 2020). This design freedom allows for a specific matching of the implant shape and mechanical properties to each individual skeletal defect, which is highly clinically relevant (Alluri et al., 2019). Integration of functionally graded open porosity into implants would allow the implant to more closely mimic the mechanical properties of human bone and its internal architecture. This mimicry of the porous structure of natural bone and its properties is essential for bone replacements (Fantini and Curto, 2017).

Despite the increasing interest and high potential of additivemanufactured porous titanium structures, commercial availability and clinical use of these types of implants is still limited. The optimization potential, mechanical properties, and biological responses of these materials have not yet been fully characterized. Previous in vitro studies focused on the demonstration of their basic biocompatibility (Xue et al., 2007; Hoffmann et al., 2014). Several animal experiments showed good integration of titanium structures with the native bone (Pattanayak et al., 2011; Van der Stok et al., 2013; Wieding et al., 2015; Shah et al., 2016). Previous studies investigated the effects of pore geometry on cell growth and differentiation (Rumpler et al., 2008; Van Bael et al., 2012). It was suggested that cell ingrowth might be enhanced by the surface curvature of voids where cell ingrowth is faster at the edges of a triangular structure than square or hexagonal structures (Rumpler et al., 2008). Effects of pore shape on bone ingrowth have been shown to be connected to the altering permeability, which may also be affected by surface curvature (Arabnejad et al., 2016; Dallago et al., 2018; Arjunan et al., 2020).

Regarding pore size, a minimum size of $100 \mu \mathrm{m}$ is found to be essential to enable the formation of blood vessels, whereas pore sizes above $300 \mu \mathrm{m}$ are supposed to enhance bone formation and vascularization (Vaccaro, 2002; Van Bael et al., 2012). Although these studies underline the huge potential of the new design freedom to generate porous structures, no consensus regarding optimal pore size/shape for cell ingrowth and bone formation has been found (Tan et al., 2017). In general, sizes of 50-700 $\mu \mathrm{m}$ were recommended (Vaccaro, 2002; Karageorgiou and Kaplan, 2005; Geetha et al., 2009; Van Bael et al., 2012). However, there are even studies suggesting that a pore size of up to $1,000 \mu \mathrm{m}$ may be beneficial for bone ingrowth (Wang et al., 2021).

Previous studies frequently focused either on the biological response or the mechanical properties of porous structures. Therefore, further knowledge about correlation among scaffold pore size and shape, mechanical properties, and cell response is essential to develop complex structures with tailored properties and functional porosity grading.

In this study, different porous $3 \mathrm{D}$ scaffolds were designed and manufactured in the titanium alloy Ti6Al4V with PBF-LB/M technology. The porosity and geometry of the structures were designed to achieve lower structural stiffness, a variety of mechanical properties and different surface curvatures to evaluate the effects of the pore shape and size on cell response. The structures were systematically evaluated regarding dimensional accuracy, mechanical properties, using compression testing and in vitro cell response, including adhesion, metabolic activity, and biological activity (alkaline phosphatase, or ALP, activity) of Saos-2 osteosarcoma cells.

The results of this work are intended to provide the basis for developing more complex porous titanium structures with various pore sizes and shapes to tailor structural mechanical properties and biological responses. This should help to match the implant to tissue properties and enable its design for a specific application.

\section{MATERIALS AND METHODS}

\section{The Design and Manufacturing of 3D Porous Structures}

To study the cell biological response in relation to specific pore characteristics such as size and shape, the investigated structures require a uniform size and shape throughout the structure. Therefore, the non-parametric approach based on smaller subunits, called unit cells, was the method of choice for the present study.

Porous structures were designed according to the design guidelines for $\mathrm{PBF}-\mathrm{LB} / \mathrm{M}$ of titanium parts; overhanging structures exceeding a critical angle of $30^{\circ}$ to perpendicular and features smaller than $200 \mu \mathrm{m}$ were avoided (Van Bael et al., 2011). To enable powder extraction throughout the structure and guarantee open porosity, a minimum pore size 


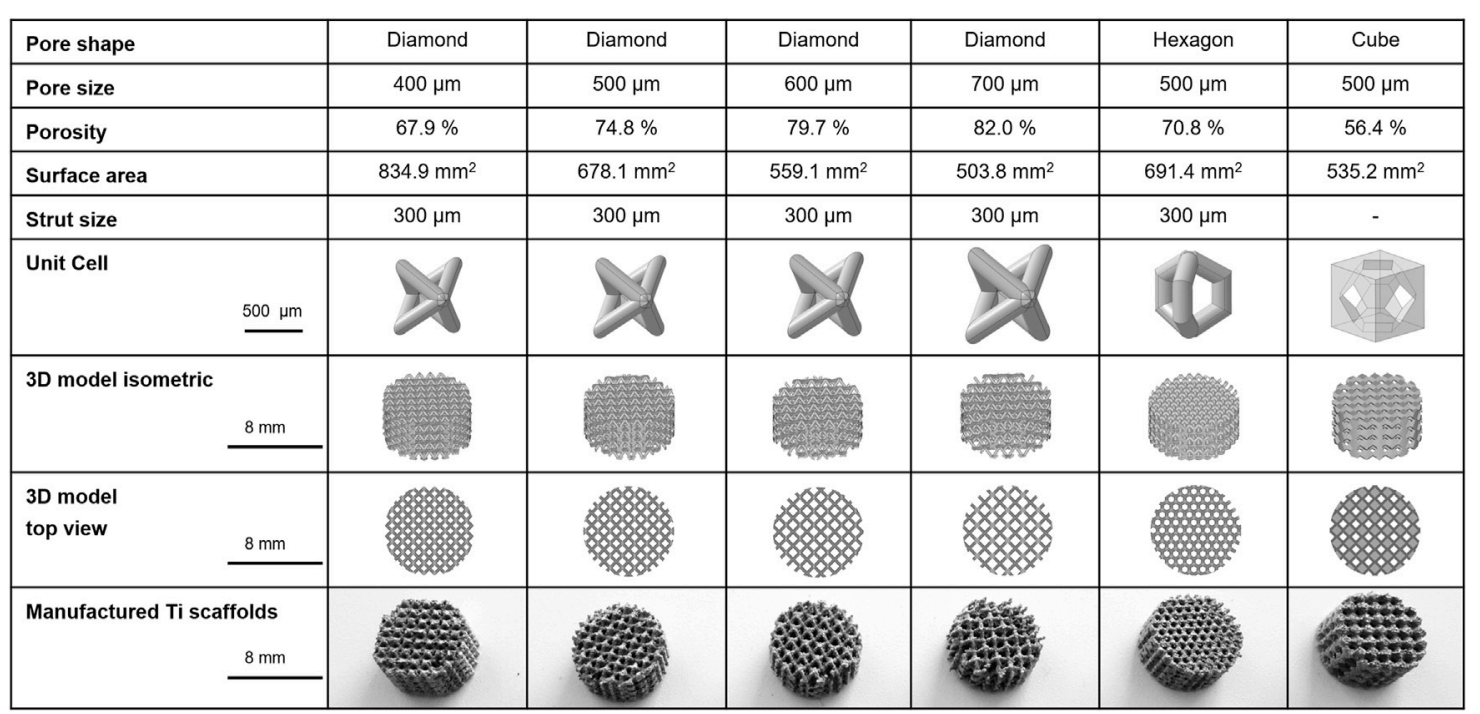

FIGURE 2 | Characteristics of 3D porous Ti6Al4V structures manufactured with PBF-LB/M technology: Structural and dimensional features of manufactured titanium scaffolds with three different pore shapes, diamond, hexagon, and cube, and pore sizes of 400, 500, 600, and $700 \mu \mathrm{m}$ (only diamond shape). Hexagon and cube scaffolds pore size was $500 \mu \mathrm{m}$.

of $400 \mu \mathrm{m}$ was used. The lattice structures were designed based on smaller subunits, called unit cells, that define the pore size and shape of the final open porous lattice structures. Three basic shapes (diamond, hexagon, and cube) with differing surface curvature and relative porosity were designed (Figure 2). These basic shapes each exhibited a uniform size and shape of interconnected pores to avoid side effects in cell biological experiments.

The Ti6Al4V structures used in this study were based on one of the following unit cell shapes and pore sizes: diamond $400 \mu \mathrm{m}$, $500 \mu \mathrm{m}, 600 \mu \mathrm{m}$ or $700 \mu \mathrm{m}$; hexagon $500 \mu \mathrm{m}$ and cube $500 \mu \mathrm{m}$ (Figure 2). The effect of the pore shape was investigated on diamond, hexagon, and cube structures at a constant pore size of $500 \mu \mathrm{m}$, whereas the effect of pore size on the cell biological response was evaluated on the diamond pore shape with $400 \mu \mathrm{m}$, $500 \mu \mathrm{m}, 600 \mu \mathrm{m}$ or $700 \mu \mathrm{m}$ pore size. The scaffold dimensions were cylindrical discs of $8 \mathrm{~mm}$ in diameter and $4 \mathrm{~mm}$ in thickness. The designed porosity of the scaffolds (Figure 2) was calculated based on the pore volume per total volume of a full material cylinder of $8 \mathrm{~mm}$ in diameter and $4 \mathrm{~mm}$ in thickness. The pore volume within the $3 \mathrm{D}$ model of the scaffolds was determined using the CAD software.

Laser powder bed fusion of metals (PBF-LB/M) is a powder bed-based additive manufacturing technology, that allows the layer-by-layer generation of complex metal parts based on a 3D model (Gibson et al., 2021). Porous Ti6Al4V structures were manufactured by PBF-LB/M using an AM 250 laser melting system (Renishaw, England). Gas atomized Ti6Al4V ELI powder (Renishaw, England) with a particle size between $15-45 \mu \mathrm{m}$ has been used as the raw material. The laser melting system was equipped with a fiber laser with a maximum power output of $200 \mathrm{~W}$ with $70 \mu \mathrm{m}$ spot size, working in pulsed mode. To avoid oxidation reactions, the

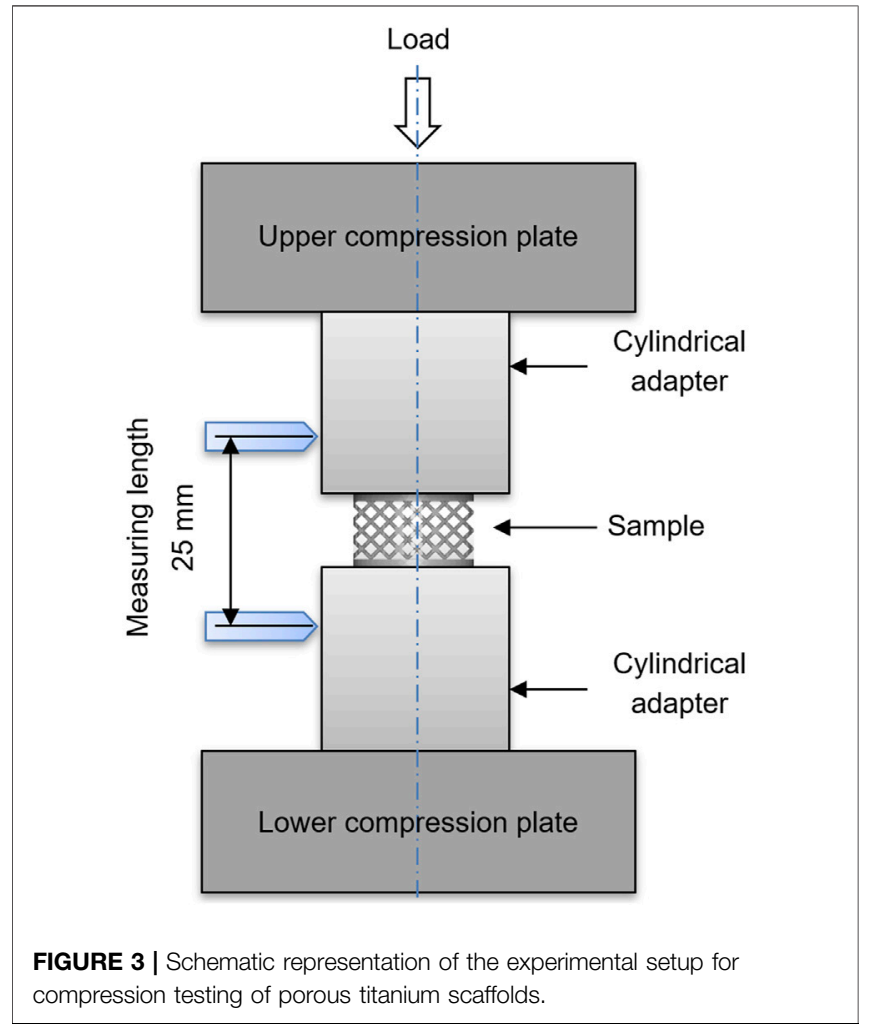

PBF-LB/M process was carried out in an argon atmosphere, where the oxygen threshold was $0.1 \%$. The porous Ti6Al4V structures were manufactured using a $30 \mu \mathrm{m}$ layer thickness, $170 \mathrm{~W}$ of laser power, $55 \mu \mathrm{m}$ point distance, $70 \mu$ s exposure, a hatch spacing of $100 \mu \mathrm{m}$ and a contour spacing of $70 \mu \mathrm{m}$. 
The produced scaffolds were cleaned in separate ultrasonic washing steps in acetone, ethanol, and twice in sterile filtered double-distilled water $\left(\mathrm{ddH}_{2} \mathrm{O}\right)$ for $15 \mathrm{~min}$ each. They were subsequently autoclaved to deactivate potential biological contamination.

The resulting effective pore sizes of additive-manufactured Ti6Al4V structures were analyzed microscopically using a Discovery.V20 Stereomicroscope (Zeiss).

\section{Compression Testing}

The structural stiffness (Young's modulus) and compression strength of scaffolds with various pore sizes and shapes were accessed by compression testing using a Z010 (Zwick-Roell) testing device. Porous structures for compression testing exhibited solid discs on both sides of the samples where compression was induced to enable a homogenous load application to the porous structure. Furthermore, cylindrical adapters with parallel faces were used to fix the MTS 632.27F20 extensometer (S/N10425111) (Figure 3). The effects of the solid discs and cylindrical adapters were compensated for using the Hooks law. Compression tests were carried out at room temperature with the application of a pre-load of $100 \mathrm{~N}$ and a compression velocity of $0.5 \mathrm{~mm} / \mathrm{min}$. Structural stiffness was defined as the slope of the elastic phase of the structure's stress-strain curves. For each type of porous structure, five specimens were tested.

\section{Static Cell Culture}

3D cell cultures were carried out using the osteosarcoma cell line Saos-2 (ATCC, Germany). The proliferation medium for Saos-2 cells was based on the basal medium DMEM-F-12/HAM (Sigma, Switzerland) supplemented with $10 \%$ fetal bovine serum (FBS, Sigma), $1 \%$ penicillin/streptomycin (Sigma), and 1\% L-glutamine (Thermo Fisher Scientific, Switzerland). The differentiation medium was additionally supplemented with $20 \mathrm{nM}$ dexamethasone (Sigma), $10 \mathrm{nM} \beta$-glycerophosphate (Sigma) and $0.4 \mathrm{mM}$ ascorbic acid (Sigma). Prior to seeding on $3 \mathrm{D}$ porous structures, the cells were expanded in $2 \mathrm{D}$ using standard cultivation procedures and kept in a humidified incubator at $5 \% \mathrm{CO}_{2}$ and $37^{\circ} \mathrm{C}$.

The evaluation of effects of pore size and shape was carried out for 21 days in 48-well plates with an initial cell seeding of $50^{\prime} 000$ Saos- 2 cells per disc scaffold. Prior to cell seeding on 3D porous structures, Ti6Al4V scaffolds were incubated with proliferation medium for $24 \mathrm{~h}$ in a humidified incubator at $5 \%$ $\mathrm{CO}_{2}$ and $37^{\circ} \mathrm{C}$.

Each scaffold was drop-seeded from all sides with cell suspension and incubated for $30 \mathrm{~min}$ at $37{ }^{\circ} \mathrm{C}$ and $5 \% \mathrm{CO}_{2}$. Following that scaffold position in the wells was changed and the cell suspension was re-seeded and incubated for another $30 \mathrm{~min}$ in order to assure the even distribution of cells in the scaffold. Finally, $1 \mathrm{ml}$ of proliferation medium was added to each well, and the scaffolds were then incubated at $37^{\circ} \mathrm{C}$ with $5 \% \mathrm{CO}_{2}$ until analyzed. The media was changed every third to fourth day. After 7 days of culture, the proliferation medium for Saos- 2 cells was changed to differentiation medium.

\section{Scanning Electron Microscopy}

At investigated time points, scaffolds were washed twice with phosphate buffered saline (PBS) (Sigma), fixed with 3\% glutaraldehyde in PBS (Sigma) for $2 \mathrm{~h}$ at $4^{\circ} \mathrm{C}$, and dehydrated in increasing concentrations of ethanol. They were then dried with hexamethyldisilazane (HMDS) (Sigma) for $24 \mathrm{~h}$ at room temperature in a fume hood and coated with gold particles using the Quorum Q150R S sputter device (Gala Instrumente GmbH, Switzerland). Finally, they were observed using an FEI Phenom Scanning Electron Microscope (Schaefer-Tec AG, Germany).

\section{PrestoBlue Cell Viability Assay}

PrestoBlue is a resazurin-based reagent to quantitatively evaluate cell viability in cell cultures (Ahmed et al., 1994; Byth et al., 2001). Viable cells convert the reagent into a strong fluorescent form, which can be detected using fluorescence measurements. The conversion and, thus, the measured signal are proportional to the number of metabolically active cells (Yu et al., 2003; Xu et al., 2015). Before each analysis, the scaffolds were placed in new wells of 24-well plates to avoid result deviation caused by cells attached to the surface of the wells during cell culture. The culture medium was replaced with the PrestoBlue reagent (Invitrogen, Switzerland) diluted 1:10 with proliferation medium for the respective cell type and samples were incubated for $2 \mathrm{~h}$ at $37^{\circ} \mathrm{C}$ with $5 \% \mathrm{CO}_{2}$. Media fluorescence was measured in the FLUOstar OPTIMA reader at $\lambda$ ex $550 \pm 10 \mathrm{~nm}$ and $\lambda$ em $590 \pm 10 \mathrm{~nm}$ (FLUOstar OPTIMA; BMG Labtech, Germany). Blanks (scaffolds without cells) were cultivated under identical conditions as seeded scaffolds. The fluorescence signal of the blanks was subtracted from the signal of seeded scaffolds as zero value correction.

\section{Quantification of Alkaline Phosphatase}

ALP is an early marker of cell differentiation into bone formation (Golub et al., 1992). Scaffolds used to measure cell viability with PrestoBlue were subsequently prepared to quantify the ALP activity. Therefore, the scaffolds were washed twice with PBS and frozen at $-80^{\circ} \mathrm{C}$ with lysis buffer to lyse the cells. The lysis buffer consisted of $150 \mathrm{mM} \mathrm{NaCl}, 10 \mathrm{mM}$ Tris, and $0.5 \%$ Triton $\mathrm{X}-100$ dissolved in $\mathrm{ddH}_{2} \mathrm{O}$ at $\mathrm{pH} 8$.

The samples were then thawed on ice and the lysis buffer was pipetted up and down inside the scaffold to lyse all cells present inside the structure. Scaffolds were then centrifuged at $10,000 \mathrm{rpm}$ for $10 \mathrm{~min}$ at $4^{\circ} \mathrm{C}$, and the supernatant was analyzed for ALP activity detection. The ALP activity was quantified based on the conversion of paranitrophenylphosphate ( $p$-NPP) to para-nitrophenol ( $\mathrm{p}-\mathrm{NP}$ ) and inorganic phosphate by ALP as previously described (Anderson, 2003). The absorption due to the resulting reaction product, $\mathrm{p}-\mathrm{NP}$, was measured by the FLUOstar OPTIMA reader at $410 \mathrm{~nm}$. The ALP activity of the samples was determined on the basis of a $\mathrm{p}$-NP standard curve.

\section{Statistical Analysis}

Data are expressed as the mean \pm standard deviation. The oneway ANOVA with the Tukey-Kramer post hoc test was used for 

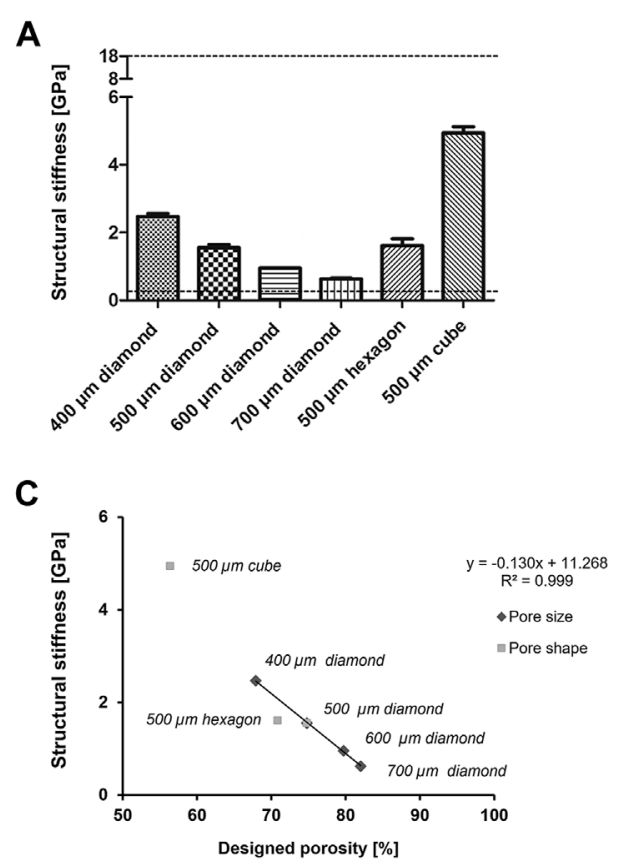

B

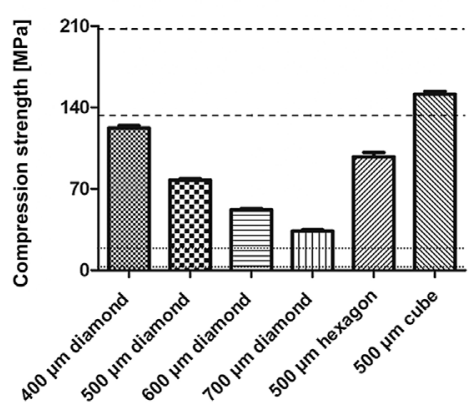

D

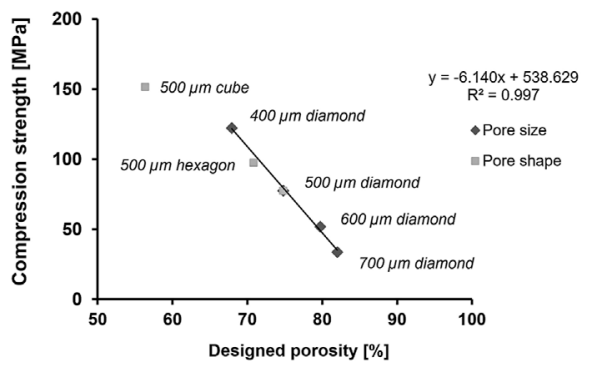

FIGURE 4 | Mechanical testing of titanium structures with various pore sizes and shapes (A) Structural stiffness and (B) compression strength of manufactured 3D titanium scaffolds, $n=5$. The dotted lines showed the fields of cortical and trabecular human bone. Correlation between the mechanical measurements [structural stiffness (C) and compression strength respectively (D)] and the designed porosity.

the statistical analysis of experimental replicates of cell cultures with a probability level of $p<0.05$.

\section{RESULTS}

\section{Design and Manufacturing of 3D Porous Structures}

Past studies investigated the effects of $3 \mathrm{D}$ printed titanium scaffold pore geometry (size or shape) on cell growth and differentiation. However, the data did not show a consensus regarding the most optimal pore shape and size combination for cell response or bone growth. In this study, 3D porous titanium scaffolds based on different pore sizes and shapes were manufactured in Ti6Al4V using PBF-LB/M technology (Figure 2).

Diamond and hexagon structures are highly porous and exhibit high surface curvature. In contrast, the cube shape was designed only with plane surfaces and edges. Its lower porosity was expected to result in higher stiffness and compression strength compared to diamond and hexagon shapes, which is reflected by the results of compression testing (Figure 4). Overall, the designed porosity of the scaffolds was between $56.4 \%$ (cube, $500 \mu \mathrm{m}$ ) and $82 \%$ (diamond, $700 \mu \mathrm{m}$ ). The designed relative porosity of the scaffolds with diamond-shaped pores increased with increasing pore size from $67.9 \%$ at $400 \mu \mathrm{m}$ up to $82 \%$ at $700 \mu \mathrm{m}$. Regarding the pore shape at $500 \mu \mathrm{m}$, the diamond shape exhibited the highest porosity of $74.8 \%$ followed by the hexagon shape with $70.8 \%$ and the cube shape with $56.4 \%$. The relative porosity of the diamond and hexagon were relatively close due to their similar strut-based design approach, compared to the cube shape. The strut diameter of diamond and hexagon shapes was designed to be $300 \mu \mathrm{m}$.

All manufactured structures showed a similar texture and high surface roughness resulting from the PBF-LB/M process and the used metal powder. Numerous powder particles were adhered to the surface, even after multiple ultrasonic cleaning steps in acetone, ethanol, and $\mathrm{ddH}_{2} \mathrm{O}$. Dimensional accuracy was dependent on the build direction, caused by rounding of the pore's edges and agglomerations of partially melted powder particles in overhanging areas (Figure 5). The deviations of the structures from the designed $3 \mathrm{D}$ model led to a slight reduction in the measured pore size compared to the designed pore size. These deviations of the designed and built structures appeared to be less pronounced with increasing pore size for the diamond-shaped structures by trend.

\section{Mechanical Properties of 3D Porous Structures}

The mechanical properties of $3 \mathrm{D}$ porous $\mathrm{Ti} 6 \mathrm{Al} 4 \mathrm{~V}$ structures were investigated by compression tests. The structural stiffness of different scaffolds was between $0.6 \mathrm{GPa}$ (diamond, $700 \mu \mathrm{m}$ ) and $4.9 \mathrm{GPa}$ (cube, $500 \mu \mathrm{m}$ ) (Figure 4A). Therefore, the stiffness increased with decreasing pore size of diamondshaped structures from $0.6 \mathrm{GPa}$ for $700 \mu \mathrm{m}$ pore size to $2.5 \mathrm{GPa}$ for $400 \mu \mathrm{m}$ pore size with all other design elements, i.e., pore shape and diameter of struts, remaining constant. 


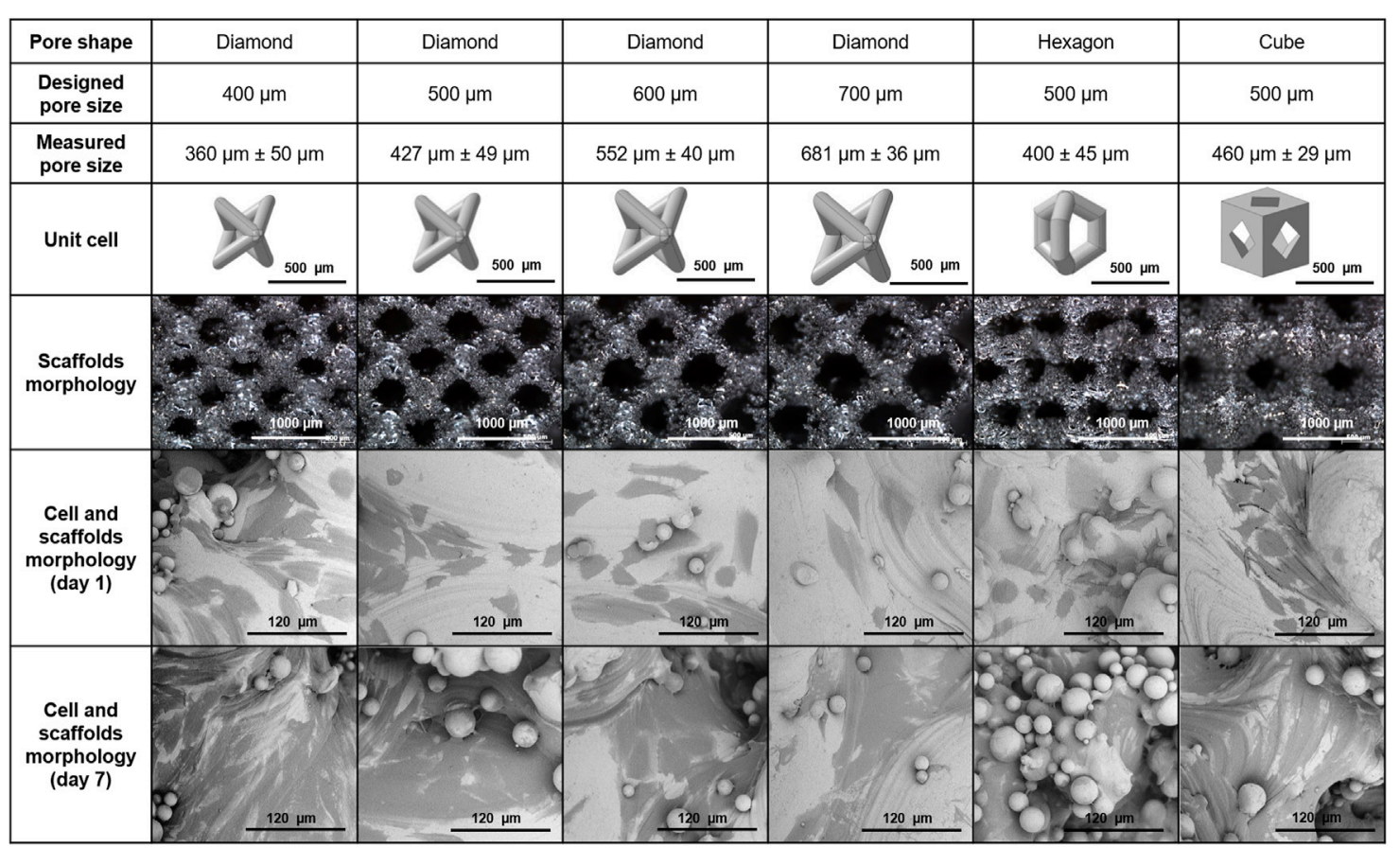

FIGURE 5 | Features of 3D porous Ti6A4V structures used in the study: pore size, shape, dimensional accuracy, 3D models of the unit cells, manufactured structures (stereo microscopy), and Saos-2 cell morphology on the scaffolds 1 day and 7 days post-seeding of the scaffolds (SEM).

Diamond- and hexagon-shaped porous structures with a $500 \mu \mathrm{m}$ pore size had similar stiffnesses of 1.6 GPa, whereas cube-shaped porous structures with the same pore size exhibited a considerably higher stiffness of $4.9 \mathrm{GPa}$. However, the hexagon shape has a lower designed porosity $(70.8 \%)$ than the diamond shape $(74.8 \%)$. There was a linear relationship between the structural stiffness and designed porosity of structures exhibiting one specific pore shape (diamond), but different pore sizes (Figure 4C). Therefore, the structural stiffness increased with decreasing designed porosity.

The compression strength of 3D porous structures increased with decreasing pore size and relative porosity and was between $34 \mathrm{MPa}$ (diamond, $700 \mu \mathrm{m}$ ) and $151 \mathrm{MPa}$ (cube, $500 \mu \mathrm{m}$ ) (Figure 4B). For the various pore sizes of the diamond shape, the compression strength increased with decreasing pore size from $34 \mathrm{MPa}$ for $700 \mu \mathrm{m}$ pore size to $122 \mathrm{MPa}$ for $400 \mu \mathrm{m}$ pore size. The cube shape exhibited the highest compression strength of $151 \mathrm{MPa}$, followed by the hexagon shape at $98 \mathrm{MPa}$ and the diamond shape at $78 \mathrm{MPa}$ at $500 \mu \mathrm{m}$ pore size. Although the cube shape had a threefold stiffness compared to the diamond and hexagon shapes, the compression strength was only factored at 1.5 or 1.9 , respectively. Therefore, the relationship between stiffness and compression strength was altered by the structure's shapes. Compression strength showed a linear relation to the designed porosity of strut-based porous structures (diamond, hexagon) (Figure 4D).

Diamond $(700 \mu \mathrm{m})$ had the lowest structural stiffness and compression strength, exhibiting the biggest pore size and highest designed porosity (Figure 4A, B). Therefore, of all the investigated structures, its properties are the closest to trabecular bone (Figure 1A). The cubic $500 \mu \mathrm{m}$ scaffold, on the other hand, exhibited the highest structural stiffness and compression strength, being the closest to the mechanical properties of cortical bone (Figure 4A, B).

\section{Cell Growth in Various Ti6Al4V Structure Morphologies}

The cell response of Saos- 2 on six different types of scaffolds (Figure 2) was accessed by investigating cell adhesion, morphology, and metabolic and ALP activity. Good cell adhesion was observed on all types of scaffolds and no apparent effect of pore size and shape on cell morphology was found. Cells tended to be aligned according to the surface texture (Figure 5). There was a notable elongation of the cells along the surface texture, whereas the cells tended to spread between and around adhered powder particles. Furthermore, layers of multiple cells were formed, particularly in areas with higher surface texture or adhered powder particles from day 7 of cell culture. This finding was primarily related to the characteristic surface texture and was independent of the structure's pore size and shape.

Cell metabolic activity increased over time, up to 21 days for all the tested types of scaffolds (Figure 6). The pore size of diamond-shaped scaffolds in the range of $400-700 \mu \mathrm{m}$ had no effect on cell metabolic activity (Figure 6A). Additionally, no significant difference in cell metabolic activity was observed among different pore shapes at most of the tested time points. On day 7 , significantly lower cell metabolic activity was measured 

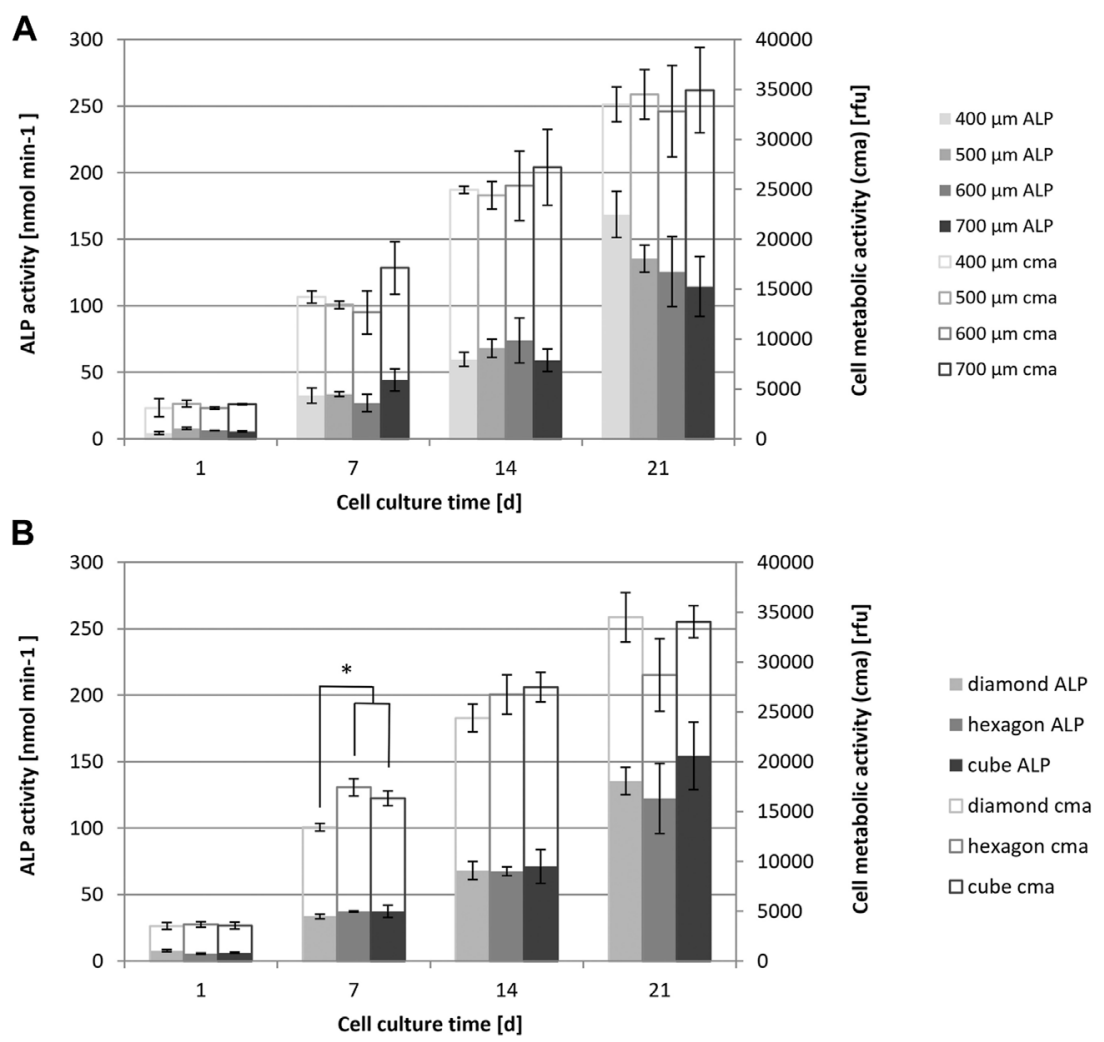

FIGURE 6 | Saos-2 cell metabolic activity (PrestoBlue assay) and ALP activity of cell cultures on scaffolds with different pore sizes (A) and shapes (B), evaluated at different time points up to 21 days of culture, $n=3$; Values are represented as mean \pm SD. *significant according to ANOVA and Tukey-Kramer post-hoc test with $p<0.05$.

for the diamond shape compared to the hexagon and cube shapes with $500 \mu \mathrm{m}$ pore size (Figure 6B). This effect was no longer present on day 14.

ALP enzyme activity was evaluated as a measure of cell differentiation and an early marker of bone formation. Overall, there was a small increase in ALP enzyme activity from day 1 to day 14 and a larger increase up to day 21 for all investigated pore sizes and shapes (Figure 6). At day 21, ALP activity was decreasing by trend with increasing scaffold pore size and was the lowest for diamond at $700 \mu \mathrm{m}$. This trend was not significant according to ANOVA analysis, as there was a relatively high variation between the experimental replicates. The variation between experimental replicates increased with increasing cell culture time. No significant effect of the diamond, hexagon or cube shape with similar pore size on the ALP activity has been noted.

\section{DISCUSSION}

In this study, the mechanical properties and biological responses of model bone osteosarcoma cells Saos-2 of additivemanufactured (Ti6Al4V) scaffolds were evaluated. The scaffolds exhibited various shapes and sizes of unit cells to reduce the structural stiffness and match better with the mechanical properties of human bone. Evaluation of pore shapes (diamond, hexagon, and cube) exhibited a wide range of structural characteristics, such as surface curvature, relative porosity, and mechanical properties. A variety of pore sizes of diamond-shaped structures with $400,500,600$, and $700 \mu \mathrm{m}$ pore diameters were evaluated to reveal effects on mechanical strength and biological responses.

The Ti6Al4V scaffolds showed minor dimensional and geometric deviations from the designed 3D model. Overall, Ti6Al4V scaffolds exhibited smaller mean pore sizes than designed (Figure 2). This finding in the context of additive manufacturing of porous structures is consistent with previous studies ( $\mathrm{Lu}$ et al., 2020). The dimensional deviations may be attributed to the aliasing effect, which is characteristic of additivemanufactured structures and even more pronounced on overhanging areas and small feature sizes (Van Bael et al., 2011).

The structural stiffness evaluated with compression testing of porous Ti6Al4V structures $(0.6-4.9 \mathrm{GPa})$ was within the range of human trabecular bone (0.3-18 GPa) (Rho et al., 1998; Morgan and Keaveny, 2001; Morgan et al., 2003; Keaveny et al., 2004). Compression strength measured $(34-151 \mathrm{MPa})$ was above the values of human trabecular bone (0.2-16 MPa) (Schoenfeld et al., 1974; Misch et al., 1999; Keaveny et al., 2004) and in the lower range of human cortical bone (130-205 MPa) (Misch et al., 1999; Keaveny et al., 2004). Despite the low structural stiffness that is 

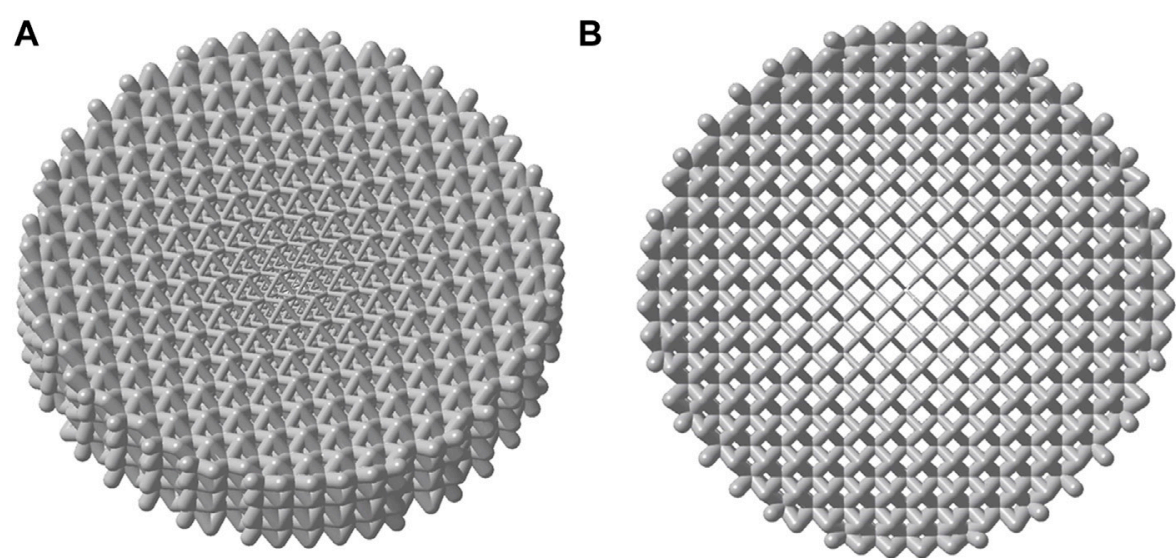

FIGURE 7 | 3D model of a porous structure with gradient porosity based on the diamond pore shape (A) isometric view (B) top view.

related to the high designed porosity, the compression strength of $3 \mathrm{D}$ porous Ti6Al4V structures is relatively high compared to human bone. This indicates that a high initial load-bearing capability may be expected for $3 \mathrm{D}$ porous Ti6Al4V structures when used in implants.

A linear relationship between structural stiffness and designed porosity was found for diamond-shaped porous structures with pore sizes between 400 and $700 \mu \mathrm{m}$. This enables prediction of structural stiffness when designing diamond-shaped porous scaffolds according to the corresponding linear regression within the tested area. As for structural stiffness, another linear relation was found between the compression strength of diamond-shaped structures and designed porosity, with pore sizes between 400 and $700 \mu \mathrm{m}$. Therefore, compression strength may be predicted based on designed porosity within the studied range of shape and size as well. Such correlations may also be found with other pore shapes and sizes.

Previous studies on scaffold pore sizes and shapes showed a correlation between the pore shape of Ti6Al4V scaffolds and cell growth and activity (Rumpler et al., 2008; Van Bael et al., 2012), whereas others found no significant pore shape effect (Markhoff et al., 2015). Present results showed no evidence of differing kinetics of cell ingrowth on porous structures with diamond, cube, or hexagon pore shapes at $500 \mu \mathrm{m}$ pore size (Figure 6).

Regarding cell adhesion and morphology, the manufacturingrelated surface texture appeared to be more decisive than the structure characteristics. Cell metabolic activity was widely independent of pore size, whereas ALP activity tended to be higher on scaffolds with smaller pore sizes after 21 days of cell culture, with diamond-shaped pores of $400 \mu \mathrm{m}$ showing the highest ALP activity value. This may indicate enhanced differentiation and may lead to enhanced bone formation on diamond-shaped scaffolds with smaller pore sizes. However, these results were not significant due to only minor differences between experimental replicas and the related impact on the statistical analysis. Additionally, the evaluation of additional markers for bone formation is required to give a better understanding of these effects. Analogous results were found in previous studies, where scaffolds of $300-500 \mu \mathrm{m}$ pore size showed enhanced bone formation and vascularization (Mullen et al., 2010; Markhoff et al., 2015). Overall, a very small effect of pore shape on ALP enzyme activity was observed. Literature showed similar results for hydroxyapatite coated titanium channels where the total amount of tissue formation was widely independent of the channel shape (Rumpler et al., 2008).

From the cell biological point of view, it may be concluded that biological responses (cell adhesion, morphology, metabolic activity, and ALP activity) are widely independent of pore shape and size within the tested range. Together with previous studies (Assad et al., 2003; Karageorgiou and Kaplan, 2005; Geetha et al., 2009; Mullen et al., 2010; Murphy et al., 2010; Fukuda et al., 2011), these findings verify the suggestion that there is no optimal pore size for cell ingrowth and bone formation, but an optimum range, which is suggested between 100 and $700 \mu \mathrm{m}$. This opens the possibility to design mechanical properties with gradient porosity without decisively affecting biological responses. However, $400 \mu \mathrm{m}$ is the minimum feasible pore size with $\mathrm{PBF}-\mathrm{LB} / \mathrm{M}$ that ensures open porosity. Smaller pores bear the risk of sintered and captured powder particles within the structure, which is expected to negatively affect vascularization and nutrient distribution. As recent literature has shown, a combination of various pore sizes could even be beneficial for bone ingrowth compared to uniform pore sizes (Wang et al., 2021).

Further work is recommended to focus on the development of additional structures in various shapes and sizes and investigation of their related effects on cell response. Topology optimization is well established in lightweight construction and is also seen as an interesting approach to customizing porous structures for medical implants. Furthermore, these structures and related cell responses need to be further investigated under loading conditions. Based on that, more complex designs of $3 \mathrm{D}$ porous Ti6AL4V structures may be developed. Functionally graded porous structures combined with an optimized dense structure should permit tailored mechanical properties of an implant to its specific application and minimal weight with full functionality (Figure 7). This gradient in porosity may achieve two things:1) low stiffness at the bone-implant interface to reduce stress 
shielding and 2) high strength of the entire implant to withstand the static and dynamic load it is subjected to during its period of application. Such a combination of multiple porosities within one implant may be applied to various implant types, not only specifically in the field of trauma. This aims to mimic hosttissue architecture and mechanical properties, matching implants to specific patients and resulting in primary stability, increased lifetime of implants, and patient satisfaction.

\section{CONCLUSION}

This study includes the evaluation of Saos-2 cell responses to 3D porous Ti6Al4V structures produced with PBF-LB/M technology. These results represent the basis for developing more complex, highly porous structures with a combination of different pore sizes and shapes with controlled structural properties. The present study concludes that cell morphology, metabolic activity, and ALP activity are widely independent of the pore shape and size within the tested range of $400-700 \mu \mathrm{m}$. Furthermore, the mechanical properties of the evaluated structures were in the range of cortical and trabecular bone. This opens the possibility to design mechanical properties with gradient porosity without decisively affecting biological responses.

The PBF-LB/M technology has been demonstrated to be a suitable method to achieve these goals and holds promise for the processing of patient-specific metallic implants to create complex architectures with controlled internal features and personalized geometry. However, at present, there are several issues that need to be addressed for these sophisticated implants to be used clinically. This includes advances in visualization and modeling techniques, more knowledge about the fatigue of such porous structures, and economic costs. At present, the

\section{REFERENCES}

Ahmed, S. A., Gogal, R. M., Jr., and Walsh, J. E. (1994). A New Rapid and Simple Non-radioactive Assay to Monitor and Determine the Proliferation of Lymphocytes: An Alternative to [3H]thymidine Incorporation Assay. J. Immunological Methods 170 (2), 211-224. doi:10.1016/0022-1759(94) 90396-4

Albrektsson, T., and Johansson, C. (2001). Osteoinduction, Osteoconduction and Osseointegration. Eur. Spine J. 10, S96-S101. doi:10.1007/s005860100282

Alluri, R., Song, X., Bougioukli, S., Pannell, W., Vakhshori, V., Sugiyama, O., et al. (2019). Regional Gene Therapy with 3D Printed Scaffolds to Heal Critical Sized Bone Defects in a Rat Model. J. Biomed. Mater. Res. 107 (10), 2174-2182. doi:10.1002/jbm.a.36727

Alvarez, K., and Nakajima, H. (2009). Metallic Scaffolds for Bone Regeneration. Materials 2, 790-832. doi:10.3390/ma2030790

Andani, M. T., Shayesteh Moghaddam, N., Haberland, C., Dean, D., Miller, M. J., and Elahinia, M. (2014). Metals for Bone Implants. Part 1. Powder Metallurgy and Implant Rendering. Acta Biomater. 10, 4058-4070. doi:10.1016/ j.actbio.2014.06.025

Anderson, H. C. (2003). Matrix Vesicles and Calcification. Curr. Rheumatol. Rep. 5, 222-226. doi:10.1007/s11926-003-0071-z

Arabnejad, S., Burnett Johnston, R., Pura, J. A., Singh, B., Tanzer, M., and Pasini, D. (2016). High-strength Porous Biomaterials for Bone Replacement: a Strategy to Assess the Interplay between Cell Morphology, Mechanical Properties, Bone cost of printing personalized implants is significantly higher than that of mass-produced alternatives, with further costs associated with the additional scans necessary to gather the required data. On the other hand, the use of customized implants has been shown to notably enhance the medical outcome of the surgery, thus potentially reducing the duration of the hospital stay and minimizing the risk of revision surgeries (Prasad et al., 2017).

\section{DATA AVAILABILITY STATEMENT}

The original contributions presented in the study are included in the article/supplementary material, further inquiries can be directed to the corresponding author.

\section{AUTHOR CONTRIBUTIONS}

All authors (LZ, KK, EB, AK, ED, and UG-H) decisively contributed to experimental design and choice of used methods. The design and manufacturing of scaffolds as well as data acquisition and evaluation were primarily carried out by LZ, supported by KK and EB, and supervised by AK, ED and UG-H. All authors contributed to the writing and reviewing of the manuscript. The figures were created by LZ and EB. AK, ED, and UG-H contributed to the project additionally with their valuable advice and experience in their fields of expertise.

\section{FUNDING}

Open access funding provided by ZHAW Zurich University of Applied Sciences.

Ingrowth and Manufacturing Constraints. Acta Biomater. 30, 345-356. doi:10.1016/j.actbio.2015.10.048

Arjunan, A., Demetriou, M., Baroutaji, A., and Wang, C. (2020). Mechanical Performance of Highly Permeable Laser Melted Ti6Al4V Bone Scaffolds. J. Mech. Behav. Biomed. Mater. 102, 103517. doi:10.1016/ j.jmbbm.2019.103517

Assad, M., Jarzem, P., Leroux, M. A., Coillard, C., Chernyshov, A. V., Charette, S., et al. (2003). Porous Titanium-Nickel for Intervertebral Fusion in a Sheep Model: Part 1. Histomorphometric and Radiological Analysis1. J. Biomed. Mater. Res. 64B, 107-120. doi:10.1002/jbm.b.10530

Ataee, A., Li, Y., Brandt, M., and Wen, C. (2018). Ultrahigh-strength Titanium Gyroid Scaffolds Manufactured by Selective Laser Melting (SLM) for Bone Implant Applications. Acta Materialia 158, 354-368. doi:10.1016/ j.actamat.2018.08.005

Byth, H.-A., Mchunu, B. I., Dubery, I. A., and Bornman, L. (2001). Assessment of a Simple, Non-toxic Alamar Blue Cell Survival Assay to Monitor Tomato Cell Viability. Phytochem. Anal. 12 (5), 340-346. doi:10.1002/pca.595

Chen, H., Han, Q., Wang, C., Liu, Y., Chen, B., and Wang, J. (2020). Porous Scaffold Design for Additive Manufacturing in Orthopedics: A Review. Front. Bioeng. Biotechnol. 8, 609. doi:10.3389/fbioe.2020.00609

Cordonnier, T., Sohier, J., Rosset, P., and Layrolle, P. (2011). Biomimetic Materials for Bone Tissue Engineering - State of the Art and Future Trends. Adv. Eng. Mater. 13, B135-B150. doi:10.1002/adem.201080098

Dallago, M., Fontanari, V., Torresani, E., Leoni, M., Pederzolli, C., Potrich, C., et al. (2018). Fatigue and Biological Properties of Ti-6Al-4V ELI Cellular Structures 
with Variously Arranged Cubic Cells Made by Selective Laser Melting. J. Mech. Behav. Biomed. Mater. 78, 381-394. doi:10.1016/j.jmbbm.2017.11.044

El-Hajje, A., Kolos, E. C., Wang, J. K., Maleksaeedi, S., He, Z., Wiria, F. E., et al. (2014). Physical and Mechanical Characterisation of 3D-Printed Porous Titanium for Biomedical Applications. J. Mater. Sci. Mater. Med. 25, 2471-2480. doi:10.1007/s10856-014-5277-2

Fantini, M., and Curto, M. (2017). Interactive Design and Manufacturing of a Voronoi-Based Biomimetic Bone Scaffold for Morphological Characterization. Int. J. Interact. Des. Manuf. 12, 585-596. doi:10.1007/s12008-017-0416-х

Fukuda, A., Takemoto, M., Saito, T., Fujibayashi, S., Neo, M., Pattanayak, D. K., et al. (2011). Osteoinduction of Porous Ti Implants with a Channel Structure Fabricated by Selective Laser Melting. Acta Biomater. 7, 2327-2336. doi:10.1016/j.actbio.2011.01.037

Geetha, M., Singh, A. K., Asokamani, R., and Gogia, A. K. (2009). Ti Based Biomaterials, the Ultimate Choice for Orthopaedic Implants - A Review. Prog. Mater. Sci. 54, 397-425. doi:10.1016/j.pmatsci.2008.06.004

Gibson, I., Rosen, D. W., Stucker, B., and Khorasani, M. (2021). Additive Manufacturing Technologies. 3rd Edition. Cham, Switzerland: Springer.

Golub, E. E., Harrison, G., Taylor, a. G., Camper, S., and Shapiro, I. M. (1992). The Role of Alkaline Phosphatase in Cartilage Mineralization. Bone Mineral. 17, 273-278. doi:10.1016/0169-6009(92)90750-8

Gugala, Z., Lindsey, R. W., and Gogolewski, S. (2007). New Approaches in the Treatment of Critical-Size Segmental Defects in Long Bones. Macromol. Symp. 253, 147-161. doi:10.1002/masy.200750722

Henkel, J., Woodruff, M. A., Epari, D. R., Steck, R., Glatt, V., Dickinson, I. C., et al. (2013). Bone Regeneration Based on Tissue Engineering Conceptions - A 21st Century Perspective. Bone Res. 1, 216-248. doi:10.4248/BR201303002

Hoffmann, W., Bormann, T., Rossi, A., Müller, B., Schumacher, R., Martin, I., et al. (2014). Rapid Prototyped Porous Nickel-Titanium Scaffolds as Bone Substitutes. J. Tissue Eng. 5, 204173141454067. doi:10.1177/2041731414540674

Karageorgiou, V., and Kaplan, D. (2005). Porosity of 3D Biomaterial Scaffolds and Osteogenesis. Biomaterials 26, 5474-5491. doi:10.1016/ j.biomaterials.2005.02.002

Keaveny, T. M., Morgan, E. F., and Yeh, O. C. (2004). "Bone Mechanics," in Standard Handbook of Biomedical Engineering and Design. Editors M. Kutz (New York: McGraw-Hill Education).

Klosterhoff, B. S., Kaiser, J., Nelson, B. D., Karipott, S. S., Ruehle, M. A., Hollister, S. J., et al. (2020). Wireless Sensor Enables Longitudinal Monitoring of Regenerative Niche Mechanics during Rehabilitation that Enhance Bone Repair. Bone 135, 115311. doi:10.1016/j.bone.2020.115311

Lewis, G. (2013). Properties of Open-Cell Porous Metals and Alloys for Orthopaedic Applications. J. Mater. Sci. Mater. Med. 24, 2293-2325. doi:10.1007/s10856-013-4998-y

Liu, T., Guessasma, S., Zhu, J., and Zhang, W. (2019). Designing Cellular Structures for Additive Manufacturing Using Voronoi-Monte Carlo Approach. Polymers 11, 1158. doi:10.3390/polym11071158

Lu, Y., Cui, Z., Cheng, L., Li, J., Yang, Z., Zhu, H., et al. (2020). Quantifying the Discrepancies in the Geometric and Mechanical Properties of the Theoretically Designed and Additively Manufactured Scaffolds. J. Mech. Behav. Biomed. Mater. 112, 104080. doi:10.1016/j.jmbbm.2020.104080

Mangano, F., Chambrone, L., Van Noort, R., Miller, C., Hatton, P., and Mangano, C. (2014). Direct Metal Laser Sintering Titanium Dental Implants: A Review of the Current Literature. Int. J. Biomater. 2014, 1-11. doi:10.1155/2014/461534

Markhoff, J., Wieding, J., Weissmann, V., Pasold, J., Jonitz-Heincke, A., and Bader, R. (2015). Influence of Different Three-Dimensional Open Porous Titanium Scaffold Designs on Human Osteoblasts Behavior in Static and Dynamic Cell Investigations. Materials 8, 5490-5507. doi:10.3390/ma8085259

Misch, C. E., Qu, Z., and Bidez, M. W. (1999). Mechanical Properties of Trabecular Bone in the Human Mandible: Implications for Dental Implant Treatment Planning and Surgical Placement. J. Oral Maxillofac. Surg. 57, 700-706. doi:10.1016/S0278-2391(99)90437-8

Morgan, E. F., Bayraktar, H. H., and Keaveny, T. M. (2003). Trabecular Bone Modulus-Density Relationships Depend on Anatomic Site. J. Biomech. 36, 897-904. doi:10.1016/S0021-9290(03)00071-X

Morgan, E. F., and Keaveny, T. M. (2001). Dependence of Yield Strain of Human Trabecular Bone on Anatomic Site. J. Biomech. 34, 569-577. doi:10.1016/ S0021-9290(01)00011-2
Mullen, L., Stamp, R. C., Fox, P., Jones, E., Ngo, C., and Sutcliffe, C. J. (2010). Selective Laser Melting: A Unit Cell Approach for the Manufacture of Porous, Titanium, Bone In-Growth Constructs, Suitable for Orthopedic Applications. II. Randomized Structures. J. Biomed. Mater. Res. 92B, 178-188. doi:10.1002/jbm.b.31504

Murphy, C. M., Haugh, M. G., and O’Brien, F. J. (2010). The Effect of Mean Pore Size on Cell Attachment, Proliferation and Migration in CollagenGlycosaminoglycan Scaffolds for Bone Tissue Engineering. Biomaterials 31, 461-466. doi:10.1016/j.biomaterials.2009.09.063

Niinomi, M. (1998). Mechanical Properties of Biomedical Titanium Alloys. Mater. Sci. Eng. A 243, 231-236. doi:10.1016/S0921-5093(97)00806-X

Parthasarathy, J., Starly, B., and Raman, S. (2011). A Design for the Additive Manufacture of Functionally Graded Porous Structures with Tailored Mechanical Properties for Biomedical Applications. J. Manufacturing Process. 13, 160-170. doi:10.1016/j.jmapro.2011.01.004

Pattanayak, D. K., Fukuda, A., Matsushita, T., Takemoto, M., Fujibayashi, S., Sasaki, K., et al. (2011). Bioactive Ti Metal Analogous to Human Cancellous Bone: Fabrication by Selective Laser Melting and Chemical Treatments. Acta Biomater. 7, 1398-1406. doi:10.1016/j.actbio.2010.09.034

Prasad, K., Bazaka, O., Chua, M., Rochford, M., Fedrick, L., Spoor, J., et al. (2017). Metallic Biomaterials: Current Challenges and Opportunities. Materials 10, 884. doi:10.3390/ma10080884

Rho, J.-Y., Kuhn-Spearing, L., and Zioupos, P. (1998). Mechanical Properties and the Hierarchical Structure of Bone. Med. Eng. Phys. 20, 92-102. doi:10.1016/ S1350-4533(98)00007-1

Rumpler, M., Woesz, A., Dunlop, J. W. C., van Dongen, J. T., and Fratzl, P. (2008). The Effect of Geometry on Three-Dimensional Tissue Growth. J. R. Soc. Interf. 5, 1173-1180. doi:10.1098/rsif.2008.0064

Schmitz, J. P., and Hollinger, J. O. (1986). The Critical Size Defect as an Experimental Model for Craniomandibulofacial Nonunions. Clin. Orthopaedics Relat. Res. 205, 299-308. doi:10.1097/00003086-198604000-00036

Schoenfeld, C. M., Lautenschlager, E. P., and Meyer, P. R. (1974). Mechanical Properties of Human Cancellous Bone in the Femoral Head. Med. Biol. Engng. 12, 313-317. doi:10.1007/BF02477797

Shah, F. A., Snis, A., Matic, A., Thomsen, P., and Palmquist, A. (2016). 3D Printed Ti6Al4V Implant Surface Promotes Bone Maturation and Retains a Higher Density of Less Aged Osteocytes at the Bone-Implant Interface. Acta Biomater. 30, 357-367. doi:10.1016/j.actbio.2015.11.013

Tan, X. P., Tan, Y. J., Chow, C. S. L., Tor, S. B., and Yeong, W. Y. (2017). Metallic Powder-Bed Based 3d Printing of Cellular Scaffolds for Orthopaedic Implants: A State-Of-The-Art Review on Manufacturing, Topological Design, Mechanical Properties and Biocompatibility. Mater. Sci. Eng. C 76, 1328-1343. doi:10.1016/ j.msec.2017.02.094

Vaccaro, A. R. (2002). The Role of the Osteoconductive Scaffold in Synthetic Bone Graft. Orthopedics 25, 571-578. doi:10.3928/0147-7447-20020502-05

Van Bael, S., Chai, Y. C., Truscello, S., Moesen, M., Kerckhofs, G., Van Oosterwyck, H., et al. (2012). The Effect of Pore Geometry on the In Vitro Biological Behavior of Human Periosteum-Derived Cells Seeded on Selective Laser-Melted Ti6Al4V Bone Scaffolds. Acta Biomater. 8, 2824-2834. doi:10.1016/j.actbio.2012.04.001

Van Bael, S., Kerckhofs, G., Moesen, M., Pyka, G., Schrooten, J., and Kruth, J. P. (2011). Micro-CT-based Improvement of Geometrical and Mechanical Controllability of Selective Laser Melted Ti6Al4V Porous Structures. Mater. Sci. Eng. A 528, 7423-7431. doi:10.1016/j.msea.2011.06.045

Van der Stok, J., Van der Jagt, O. P., Amin Yavari, S., De Haas, M. F. P., Waarsing, J. H., Jahr, H., et al. (2013). Selective Laser Melting-Produced Porous Titanium Scaffolds Regenerate Bone in Critical Size Cortical Bone Defects. J. Orthop. Res. 31, 792-799. doi:10.1002/jor.22293

Wang, C., Xu, D., Lin, L., Li, S., Hou, W., He, Y., et al. (2021). Corrigendum to "Large-Pore-Size Ti6Al4V Scaffolds with Different Pore Structures for Vascularized Bone Regeneration". Mater. Sci. Eng. C. 131, 112531. doi:10.1016/j.msec.2021.112531

Wieding, J., Lindner, T., Bergschmidt, P., and Bader, R. (2015). Biomechanical Stability of Novel Mechanically Adapted Open-Porous Titanium Scaffolds in Metatarsal Bone Defects of Sheep. Biomaterials 46, 35-47. doi:10.1016/ j.biomaterials.2014.12.010

Xu, M., McCanna, D. J., and Sivak, J. G. (2015). Use of the Viability Reagent PrestoBlue in Comparison with alamarBlue and MTT to Assess the Viability of Human Corneal Epithelial Cells. J. Pharmacol. Toxicol. Methods 71, 1-7. doi:10.1016/j.vascn.2014.11.003 
Xue, W., Krishna, B. V., Bandyopadhyay, A., and Bose, S. (2007). Processing and Biocompatibility Evaluation of laser processed porous titanium. Acta Biomaterialia 3, 1007-1018. doi:10.1016/j.actbio.2007.05.009

Yu, H. G., Chung, H., Yu, Y. S., Seo, J. M., and Heo, J. W. (2003). A New Rapid and Non-radioactive Assay for Monitoring and Determining the Proliferation of Retinal Pigment Epithelial Cells. Korean J. Ophthalmol. 17 (1), 29-34. doi:10.3341/kjo.2003.17.1.29

Conflict of Interest: Author ED was employed by Nobel Biocare.

The remaining authors declare that the research was conducted in the absence of any commercial or financial relationships that could be construed as a potential conflict of interest.
Publisher's Note: All claims expressed in this article are solely those of the authors and do not necessarily represent those of their affiliated organizations, or those of the publisher, the editors and the reviewers. Any product that may be evaluated in this article, or claim that may be made by its manufacturer, is not guaranteed or endorsed by the publisher.

Copyright (c) 2022 Zumofen, Kopanska, Bono, Kirchheim, De Haller and GrafHausner. This is an open-access article distributed under the terms of the Creative Commons Attribution License (CC BY). The use, distribution or reproduction in other forums is permitted, provided the original author(s) and the copyright owner(s) are credited and that the original publication in this journal is cited, in accordance with accepted academic practice. No use, distribution or reproduction is permitted which does not comply with these terms. 